\title{
Ontology modeling for generation of clinical pathways
}

\author{
Jasmine Tehrani ${ }^{1}$, Kecheng Liu ${ }^{1,2}$, Vaughan Michel $^{1}$ \\ ${ }^{1}$ Informatics Research Centre, University of Reading (United Kingdom) \\ ${ }^{2}$ School of Information Management and Engineering, Shanghai University of Finance and Economics (China) \\ g.tehrani@pgr.reading.ac.uk,.k.liu@,reading.ac.uk,v.a.michel@,reading.ac.uk
}

Received June 2011

Accepted February 2012

\section{Abstract:}

Purpose: Increasing costs of health care, fuelled by demand for high quality, cost-effective healthcare has drove hospitals to streamline their patient care delivery systems. One such systematic approach is the adaptation of Clinical Pathways (CP) as a tool to increase the quality of healthcare delivery. However, most organizations still rely on are paper-based pathway guidelines or specifications, which have limitations in process management and as a result can influence patient safety outcomes. In this paper, we present a method for generating clinical pathways based on organizational semiotics by capturing knowledge from syntactic, semantic and pragmatic to social level.

Design/methodology/approach: The proposed modeling approach to generation of CPs adopts organizational semiotics and enables the generation of semantically rich representation of CP knowledge. Semantic Analysis Method (SAM) is applied to explicitly represent the semantics of the concepts, their relationships and patterns of behavior in terms of an ontology chart. Norm Analysis Method (NAM) is adopted to identify and formally specify patterns of behavior and rules that govern the actions identified on the ontology chart. Information collected during semantic and norm analysis is integrated to guide the generation of CPs using best practice represented in BPMN thus enabling the automation of CP.

Findings: This research confirms the necessity of taking into consideration social aspects in designing information systems and automating CP. The complexity of healthcare processes can 
be best tackled by analyzing stakeholders, which we treat as social agents, their goals and patterns of action within the agent network.

Originality/value: The current modeling methods describe CPs from a structural aspect comprising activities, properties and interrelationships. However, these methods lack a mechanism to describe possible patterns of human behavior and the conditions under which the behavior will occur. To overcome this weakness, a semiotic approach to generation of clinical pathway is introduced. The CP generated from SAM together with norms will enrich the knowledge representation of the domain through ontology modeling, which allows the recognition of human responsibilities and obligations and more importantly, the ultimate power of decision making in exceptional circumstances.

Keywords: Clinical pathways, process modeling, BPMN, organizational semiotics, ontology chart, semantic analysis method, medical quality improvement

\section{Introduction}

One of the main challenges in healthcare domain is to increase the treatment quality. In order to achieve this goal, hospitals need to utilize standardized clinical protocols used in many domains of medicine. Such a protocol contains detailed medical plans for diagnosis, therapy scheme and follow-up. Moreover, it encloses the information required so as to deal with exceptional situations, which occur during the treatment execution time and require quick and appropriate modifications of the treatment of a patient, thus increasing the flexibility of the treatment processes. One valuable tool to achieve the above-mentioned objectives is "Clinical Pathways".

Clinical pathway (CP) is developed as a standard way to manage medical activities since the 1980's. CPs are structured multi-disciplinary care plans or medical processes in which diagnostic and therapeutic interventions performed for a particular diagnosis are described sequentially (Audimoolam, Nair, Gaikwad \& Qing, 2005).

However, the application and adaptation of CP in a local hospital setting, inevitably has some limitations of process management in practice. Despite the benefits, there are many instances which show that CP fails to offer a clear description of activities, conditions, sequence and authorities of action of a care process (Carthey, 2010). The conditions and consequences expressed in CP are always bound and there is no capability to handle human discretion. As a result, current application of $\mathrm{CP}$ cannot handle situations where decisions are made solely on human judgment. Hence, one cannot model dynamics of care activity and alternative 
procedures in which decision are made solely based on human judgment (Liu, Sun, Barjis \& Dietz, 2003). This issue is mostly related to healthcare settings where processes are complex, less structured and are made up of social agents such as physicians, departments with goals that they actively pursue in constant interaction with a network of other social agents (Mould, Bowers \& Ghattas, 2010). In this dynamic network of interconnected activities, the current adaptation of CP becomes a source of patient safety incident (Mould et al., 2010). Viewing errors as the result of poorly designed systems more so than incompetent or misguided individuals introduces variables that operationalize dynamics seen at process management levels (Forster, 2003, Corrigan, 2005). Workflow management has been cited as potentially important in addressing medical errors and patient safety in many publications like the "To Err Is Human" and "Crossing the Quality Chasm" (Corrigan, 2005). Such publications place at the core of a successful systems-based approach to reducing error, the need for a strong patientsafety culture, simplified process design, development of clear work flow of activities and use of patient-centric modeling approaches in adaptation of CP to local settings (Forster, 2003, Audimoolam et al., 2005) .

A possible approach to improve $\mathrm{CP}$ and as a result reduce patient safety incidents is to use organizational semiotics methods to capture and represent the CP knowledge to determine the underlying semantics of $\mathrm{CP}$ and the relationship between the human agents and their patterns of behavior. We use Norm Analysis Method (NAM) to extracts and analyze patterns of care activities. Based on the information captured during semantic analysis and norm analysis, a process oriented analysis is conducted. Business Process Modeling Notation (BPMN) is used to model the CP and a socio-technical method to map informal safety norms in to process models.

\section{Clinical Pathways}

Clinical pathways (CP), also known as care pathways, critical pathways, integrated care pathways, or care maps, are one of the main tools used to manage the quality in healthcare concerning the standardization of care processes. It is proven that their implementation reduces the variability in clinical practice and improves outcomes (Cabitza et al., 2008).

Generally, clinical pathways refer to medical guidelines. However a single pathway may refer to guidelines on several topics in a well specified context. CP is a multidisciplinary management tool based on evidence-based practice for a specific group of patients with a predictable clinical course, in which the different tasks (interventions) by the professionals involved in the patient care are defined, optimized and sequenced either by hour or (acute care) or visit (homecare). Outcomes are tied to specific interventions (Mould et al., 2010). Clinical pathways (integrated care pathways) can be seen as an application of process management thinking to the improvement of patient healthcare. A care pathway is typically 
crystallised in the development and use of a single all-encompassing bedside document, that will stand as an indicator of the care a patient is likely to be provided in the course of the pathway going forward; and ultimately as a single unified legal record of the care the patient has received, and the progress of their condition, as the pathway has been undertaken (Audimoolam et al., 2005).

\subsection{Paper-based pathway guidelines}

Despite the widespread introduction of information technology into primary health care within the United Kingdom, medical practitioners continue to use the more traditional paper medical record often alongside the computerized system. The result of our study in one of the largest UK health trusts shows that clinical pathways are implemented as paper-based pathway specifications which act as guidelines by providing advisory knowledge about actions and reference information before the event. They are also a means of ensuring the right work was done via control norms and capturing precepts and evaluations of clinical conditions in terms of tables and forms to be completed by the nurse. Clinical notes of what work was done both in terms of the action taken and the patient status provided new knowledge and beliefs/facts for later clinical decision making and actions. A CP can be considered as the nearest clinical document to a process map for a specific clinical intervention.

Subsequently, the paper-based implementation of CPs as pathway specifications cannot handle situations where decisions are made solely on human judgment (West, Guthrie, Dawson, Borrill \& Carter, 2006). Hence, one cannot model dynamics of care activity and alternative procedures in which decision are made solely based on human judgment (Liu et al., 2003). This issue is mostly related to healthcare settings where processes are complex, less structured and are made up of social agents such as physicians, departments with goals that they actively pursue in constant interaction with a network of other social agents (Mould et al., 2010). In this dynamic network of interrelated activities, the current adaptation of CGs becomes a source of patient safety incident (Carthey, 2010).

\subsection{Related work of clinical pathway modeling}

A considerable amount of literature has been published on the modeling of clinical pathways. However, the current research on clinical pathways inevitably has some limitations which lead to patient safety incidents due to incomplete knowledge conceptualization of domain. Abidi and Chen (2006) present a semantic web framework and identified knowledge types that directly impact care planning. More recently, different methods are proposed to represent the clinical guidelines which are originally paper based as Computer Interpretable Guidelines, (CIG) most of which can be visualized in flowcharts (Sonnenberg \& Hagerty, 2006). Dijerome designs the linear sequential model for computerized clinical pathway (DiJerome, 1992). However, as their CP capability was in the form of checklist and consequently very limited, sate-transition model 
for electronic care map is developed (Okada, Ohboshi, Kuroda, Nagase \& Yoshihara, 2005). Plege et al. conducted a critical analysis of a number of CIG modeling methodologies and established a consensus on the common structure (Mor Plege, 2012). These are close to the notion of CPs adopted in this paper. Other authors represented clinical pathway knowledge as a clinical pathway ontology which offers a detailed ontological model describing the structure and function of clinical pathways (Hurley \& Abidi, 2007).

These methods describe clinical pathways mainly from a structural aspect and describe the interrelationship of activities in CP. As a result, there is a lack of a robust mechanism for describing possible patterns of human behavior and decision-making models. Therefore, there is gap between CP knowledge and its implementation, which may lead to patient safety incident. To this end, in this paper, we introduce a semiotic approach and its associated methods mainly Semantic Analysis Method (SAM) and Norm Analysis Method (NAM) to model the $\mathrm{CP}$ knowledge and hence enable the generation of clinical pathway. The clinical pathway generated from SAM together with norms semantically enriches the knowledge represented for the domain by enabling the analysis of patterns of behaviors of various agents involved in care process. The possible pattern of behavior are analyzed using NAM which gives a means to formally specify the general patterns of behavior, their condition and temporality explicitly (Wright, 1963). This enables one to predict and anticipate their actions and hence co-ordinate with other agents. Furthermore, the appropriate selection and structure of clinical activities based on ever changing patient condition is ensured by mapping informal/safety norms in clinical pathways.

\section{Ontology modeling for generation of clinical pathways}

The proposed ontology-based modeling approach for generation of clinical pathways, adopts organizational semiotics methods including Semantic Analysis Method (SAM) and Norm Analysis Method (NAM) to explicitly represent the semantics of the concepts and their relationships, patterns of behavior and norms governing the action taken. Human factors are believed to contribute to at least $40 \%$ of safety errors, therefore in order to improve patient safety it is necessary to capture and represent informal/safety factors in CPs. SAM gives us a basis for analysing human/Informal factors in healthcare setting by providing the method for identifying and representing authorities of action and their pattern of behavior. In following sections, we will discuss the base methods and techniques used in this approach including, SAM and NAM and BPMN. This is followed by a detailed review methodology including a definition of the stages of the methodology.

\subsection{Base methods}

The core methods and techniques adopted in this methodology are discussed in order to provide a background. 


\section{Organizational semiotics (OS)}

Organizational semiotics, studies the nature, characteristics, function and effect of information and communication within organizational contexts. An organization is defined as a social system in which agents behave in an organized manner conforming to a certain system of norms. These norms are regularities of perception, behavior, belief and value that are expressed as customs, habits, patterns of behavior and other cultural artifacts (Stamper, 2009).

In healthcare environment, some of the clinical and organizational functions are of high regularity and have rules that can be clearly formalized. Within the formal part of the actions, a fraction of these may be very repetitive and can be automated by computer-based systems. There are a set of methods to support the use of OS concepts for modeling information systems, named MEASUR - Methods for Eliciting, Analyzing and Specifying Users' Requirement (Stamper, 2001). In this research, Semantic Analysis Method (SAM) and Norm Analysis Method (NAM) are adopted to explicitly represent the semantics of the concepts and their relationships, patterns of behavior and norms governing the action taken.

\section{Semantic Analysis Method}

We believe that given the complexity of healthcare processes and the unique nature of healthcare settings which is comprised of different agents each perusing individual's goals; therefore it is crucial to have a semantically rich description of clinical activities. Consequently, we take a healthcare knowledge management approach to represent and conceptualize CP knowledge. SAM, from OS is adopted to conduct a semantic analysis in order to develop a semantic representation of CP in terms of clinically-oriented CP ontology chart.

\section{Norm Analysis Method}

This is followed by norm analysis to identify rules that govern clinician's patterns of activities within CP. Additionally, NAM provides a robust systemic approach to capture and formally represent human/informal factors that affect patient safety outcomes. This is central to our approach since human factors are believed to contribute to at least $40 \%$ of safety errors. Consequently, in order to achieve significant improvements in patient safety results, it is necessary to capture and represent informal/safety factors in CPs.

\section{BPMN modeling technique}

The modeling framework presented in this paper uses Business Process Modeling Notation (BPMN) to model the complex activities in CP (Dadam et al., 1997, Indulska et al., 2009). In order to generate clinically operate able $\mathrm{CP}$, it is essential to view CPs as process workflows. Despite the large amount of research in the area of CP modeling, the design and optimization 
of $\mathrm{CP}$ is not yet well establishes in hospitals and as a result, there is not yet a standard and formalised approach towards the representation of CP in healthcare setting. To overcome this weakness, in this paper we are representing a case for exploring the potentials of BPMN to design standardized CPs that are executable using workflow execution engines. BPMN techniques are adopted to enable the precise identification and representation operational aspect of CPs including roles, responsibilities, agents, decision points and sequence of CP.

The principal concept of ontology modeling for generation of $\mathrm{CP}$ is to exploit the computerized $\mathrm{CP}$ in terms of ontology charts to generate CPs based on workflow modeling techniques. Organization semiotics methods including SAM and NAM are adopted to develop a semantically rich representation of clinical activities within healthcare domain.

\subsection{The process of automatic CP generation}

The key goal is to exploit the computerized CP in terms of ontology charts to generate CPs based on workflow modeling techniques. Organization semiotics methods including SAM and NAM are adopted to develop a semantically rich representation of clinical activities within healthcare setting. This is carried out in three main steps as discussed below.

\section{Step 1: Knowledge representation}

A healthcare knowledge management approach is taken to represent knowledge of pathway specifications in form of an ontology chart that conceptualizes the knowledge in terms of possible patterns of behaviors, authorities of action, semantic units and their relationships.

\section{Step 2: Business dynamics analysis using norms}

Norms exist in a community and will govern how members behave, think, make judgments and perceive the world (Stamper, 2009). In an organisation, norms reflect regularities in the behavior of members allowing co-ordination of their actions. Norms are developed through practical experiences of agents in a society and in turn have functions of directing, coordinating and controlling actions within society (Liu, 2000). The function of norm is to determine whether patterns of behavior are lawful or acceptable in the context of the society. Norm analysis of domain, allows one to predict and anticipate actions and hence co-ordinate with other agents. Norm analysis method is introduced to identify rules that govern the actions identified on the ontology chart. Norms, in addition to the knowledge already identified in sematic analysis and represented using ontology chart, specify the details of these possible behaviors. The final activity of this stage is transformation of CGs along with the knowledge captured during SAM and NAM to process models using BPMN standards. 


\section{Step 3: Mapping the ontology chart to BPMN}

Towards modeling CP knowledge as workflow elements, since BPMN, by itself does not provide any formal semantics for its constructs, the knowledge represented during semantic and norm analyses are integrated in order to guide the generation of clinical pathways in form of workflow models.

\section{Ontology-based domain knowledge representation}

The step one towards automatic CP generation is knowledge representation. It involves the abstraction of domain-specific knowledge in terms of concepts that encapsulate the domain knowledge, problem-solving behavior, operational processes, and functional constraints. In healthcare, domain-specific knowledge is captured in terms of clinical practice guidelines, clinical pathways and research articles. For the purpose of this research, the major gynecology $\mathrm{CP}$ served as the domain knowledge artifacts. We adopted a knowledge management approach, in particular the use of Semantic Analysis to produce an ontology model of the problem domain. Our major gynecology ontology captures the salient diagnostic, treatment and operational concepts, and relates these concepts using semantic and pragmatic relationships to form a gynecology surgery knowledge model. Organizational semiotics methods including Semantic Analysis Method (SAM) and Norm Analysis Method (NAM) are adopted to explicitly represent the semantics of the concepts and their relationships, patterns of behaviour and norms governing the action taken. SAM gives us a basis for analysing human factors in healthcare setting by providing the method for identifying and representing authorities of action and their pattern of behaviour.

\subsection{Semiotic approach to ontology modeling}

Ontology is defined as a conceptual, shared and explicit specification of concept in a domain and the relationships among them (Gruber, 1995). Ontology form the organizational semiotics perspective conceptualizes knowledge of the problem domain represented by ontologically related semantic units. The semantic analysis method (SAM) is a method for conceptualizing knowledge of a problem domain and to analyze the patterns of behaviors of various agents (i.e., physician or doctors) in an organization (Liu \& Ong, 1999). Semantic analysis consists of a negotiated understanding, between relevant process intervenients, of the meanings of domain specific signs, including agents, concepts, relationships, and other related aspects. The result of semantic analysis is provided in a graphical format, using what is called 'ontology chart', as shown in in figure 1. The ontology chart models concepts and the responsible authorities of actions in the problem domain and captures domain knowledge supported by semantic units and ontological dependencies (Liu, 2001). In this way, we can define the possible patterns of medical behaviour involved in CP such as operation and discharge and the people who perform these behaviours such as physicians or recovery nurses and the relationship between them as 
behaviours. Fig. 1 is the result of SAM conducted for a major gynecology surgery guideline. An ontology chart introduces concepts as semantic units representing an agent, affordance or role label. An affordance is a possible pattern of behaviour available to a member of society, represented as rectangles. The affordance on the right is ontologically dependent on the one on left. This is called ontological dependency. For example, the antecedent Recovery nurse affords the 'evaluate rehabilitation status' behaviour. A role label is associated with an agent that undertakes some specific responsibilities. A role is always defined with respect to the role carrier on its left and the concept determining the responsibility on its right (Liu, 2001). An agent may have several roles. For example, when a person works in hospital, his role is staff; when a person is hospitalized in hospital, his role is patient. In addition, a concept may have properties prefixed by the hash sign (\#) that capture descriptive information about the concept. For example, the concept of Ward has the properties of \#ward name and \#ward number in the following charts which are called determiners. Agents are represented as ellipses and represent those who can take responsibility for their actions. It could be an individual, a group of people e.g., society, hospital and person. A dependent concept, its immediate antecedent concepts and the ontological dependencies between them form a semantic context within which the behavioural patterns of the dependent concepts are defined. In addition, the ontology model can describe the generic-specific relationships. In Figure 1 for example, under the generic heading of staff, more specific items can be found: AHP, Doctor and nurse. Through SAM, possible patterns of behaviours in CP and their relationships are represented in an ontology chart which delineates the boundary of concern in the analysis and defines the meaning of terminology used in the clinical pathway model. Thus, misunderstanding caused by interdisciplinary communications is avoided. For a complete description of the syntax of ontology charts, reader may consult.

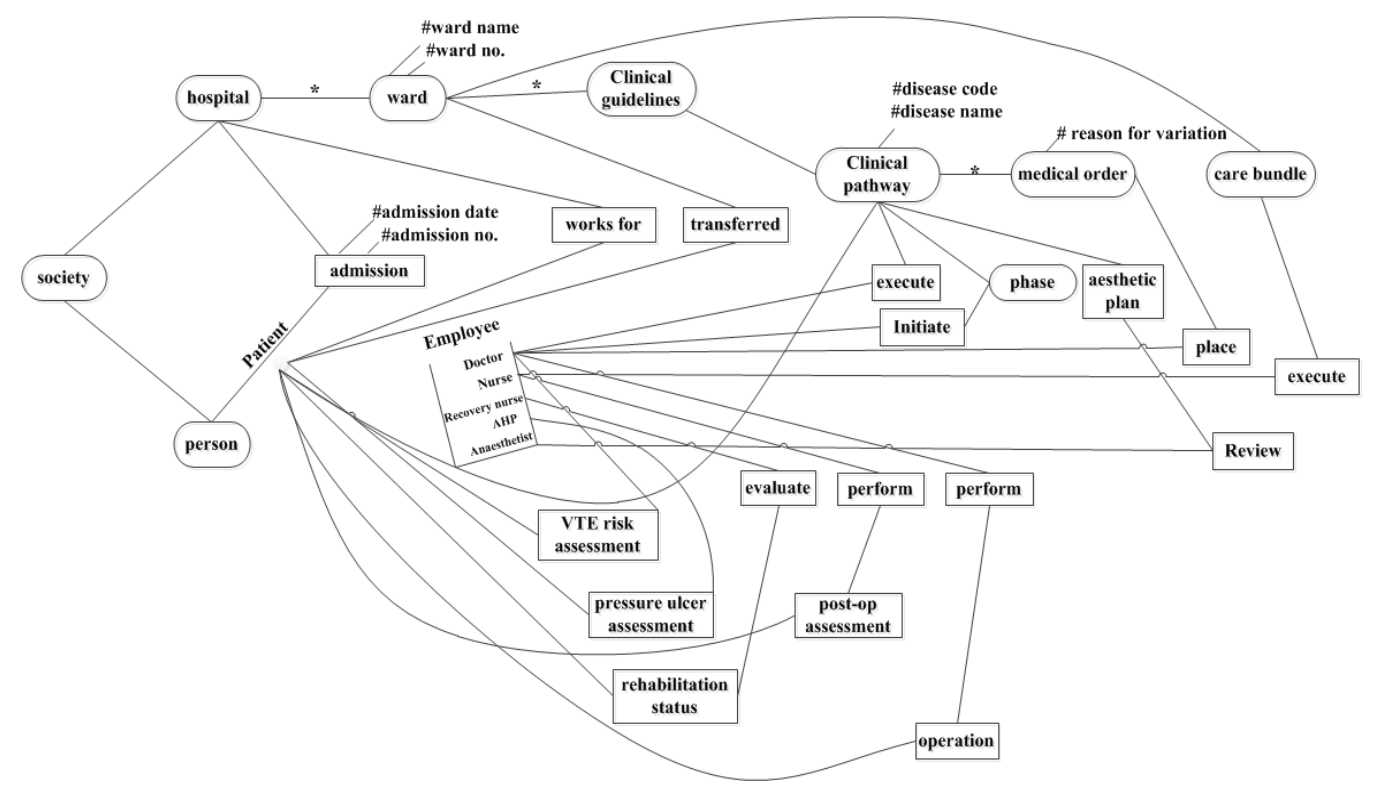

Figure 1. Ontology chart representing major gynecology care pathway 


\section{Analysis of business dynamics using norms}

The second step in automatic CP generation is to analyze business dynamics using norms. Norm Analysis Method (NAM) is introduced to identify rules that govern the actions identified on the ontology chart by determining whether certain patterns of behavior are acceptable in the problem domain. Norm analysis, in addition to knowledge captured during semantic analysis, enriches the domain analysis by capturing details of the possible patterns of behaviors; e.g., the conditions where certain clinical actions must happen or where they are actually impossible. The patterns of behavior entailed in ontology chart are formally specified by norms. These are the constraints and rules an affordance must act upon during its existence as depicted in the ontological chart. NAM provides a systematic approach a method to identify and represent the norms governing the actions of agents identified in healthcare setting by determining whether certain patterns of behaviours are acceptable or legal in the problem domain.

Norm specification uses the information collected in the previous stages to develop a formal specification of the norms. The norms are described as the following format (Liu, 2000):

Whenever <condition > If < state> Then <agent > Is < deontic operator > To < action>

"Condition" and "state" are defined from the determined pre-conditions. The precondition for one of the norms in this case of affordance "Execution" being that "the patient is hospitalized for treatment", this makes up the whenever part of the norm. A second pre-condition determines the state part i.e. "if there is a risk of bleeding", "if surgery is delayed for more than 14 days" which are usually indicated in trigger analysis. "Agent", as an authority, is responsible for executing defined action(s) or allocating them to other agents which is indicated in responsibility analysis. The entitlement is defined by the Deontic operators. "Deontic operator" is derived from Deontic Logic and can be one of the following: "obliged", "permitted" and "prohibited" which prescribe what people must, may, and must not do. It is essential to recognize that norms are not as rigid as logical conditions therefore they allow to model situations were decisions are made solely based on human judgment and there is a degree of flexibility in patterns of behavior. For those actions that are "permitted", whether the agent will take an action or not is seldom deterministic. This elasticity characterizes the clinical processes and allows the subjective activity, therefore is of particularly value to support personalized clinical pathway. Table 2 shows a list of norms for the control conditions of the care process in major gynecology $\mathrm{CP}$. 


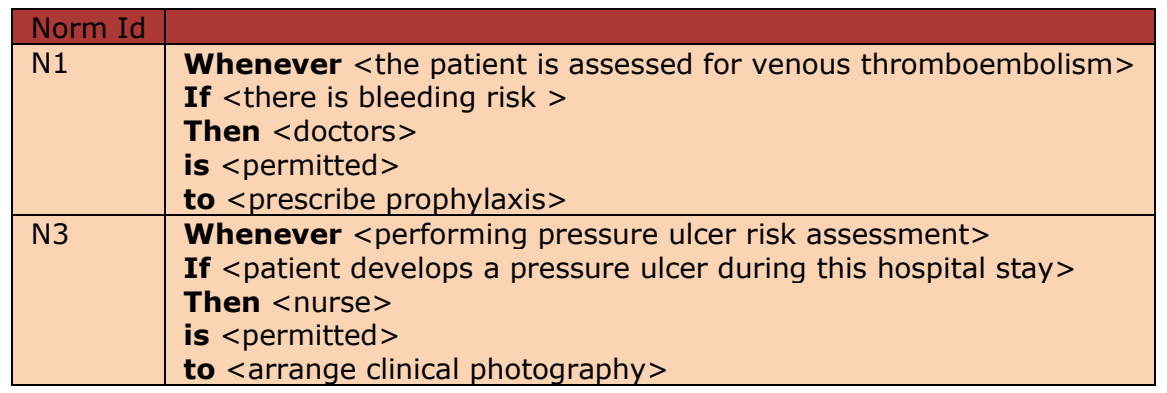

Table 1 . Norm analysis for major gynecology care surgery

\section{Generating clinical pathways from the ontology model}

As the third step in automatic CP generation, BPMN is adopted to model patient care delivery (Muthu, Whitman \& Cheraghi, 2006). For this purpose, clinical guidelines and paper-based pathway specifications are transformed into normative business process models which can address social and human factors affecting patient safety and enables identification and modeling of temporal aspects of care delivery by integrating the results of SAM and NAM into process models. Using BMPN modeling techniques, a graphical representation of clinical workflows are represented (Aguilar-Saven, 2004). Consequently, it will become easier for both medical experts and business process modelers to identify and model complex workflows. The final stage of proposed methodology is to model the CP knowledge as a workflow model whilst maintaining its medical integrity.

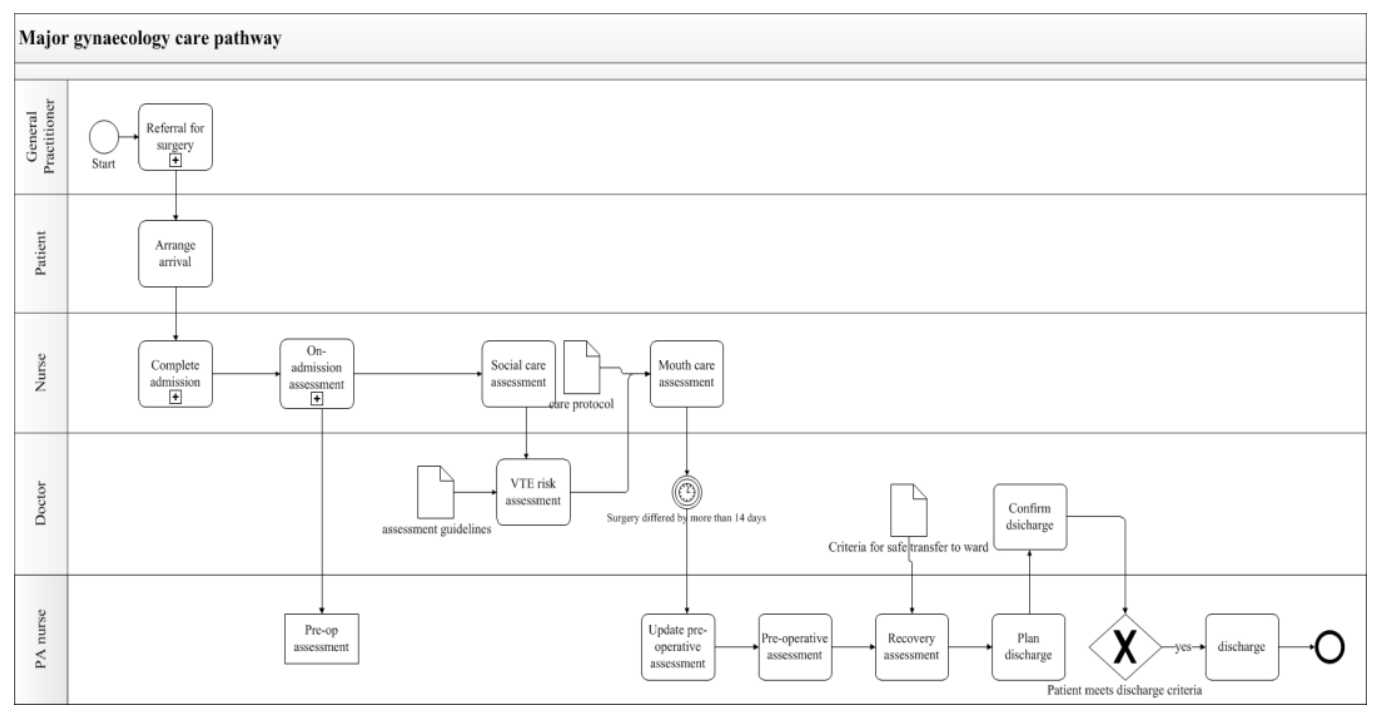

Figure 2. Part of CP for major gynecology surgery

BPMN is adopted since it gives a rational means of organizing information that is processed to perform care activities (Staccini et al., 2005; Aguilar-Saven, 2004). In addition, they enable modeling of the complete end-to-end activities which results in a clear and consistent workflow model (Becker \& Janiesch, 2008). This improves interdisciplinary communication. Process modeling also facilitates the integration of information systems with the dynamics of a 
$\mathrm{CP}$ in terms of action, roles and data exchange. Figure 2 shows part of a Major Gynecology pathway modeled using BPMN techniques. During the mapping process, it is critical to maintain the clinical integrity of the $\mathrm{CP}$ and yet enrich the knowledge representation using BPMN techniques thus achieving a CP symbolized using BPMN. To this end, the knowledge captured during SAM and NAM are integrated to guide the modeling of CP. The result of NAM is integrated in CP by incorporating norms into the business process diagram. In the diagram, each control condition is labelled as [N\#] where \# is the number for identification. The labels are then elaborated in the norm specifications to indicate the condition, the actor and action to be undertaken. Figure 3 depicts the extended care process for applying water low pressure ulcer assessment.

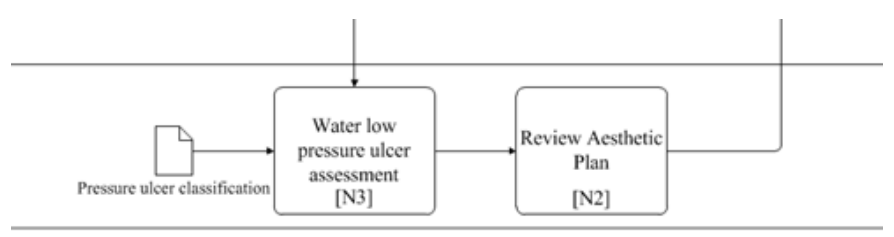

Figure 3. Extension of clinical pathway for major gynaecology surgery with norm

\section{Discussion and conclusion}

This research offers a solution for CP modeling using organisational semiotics methods and techniques. The proposed framework addresses social and informal/safety factors which conspire together to influence the outcome of patient safety. To this end, a semiotics-oriented method which adopts organisational semiotics methods, in particular, SAM and NAM is proposed. Semantic analysis method is applied to explicitly represent the semantics of the concepts, their relationships and patterns of behavior which offered a basis for analysing human/Informal factors in healthcare setting. The proposed approach enables the transformation of clinical guidelines and paper-based pathway specification to executable workflow models.

The information collected during semantic analysis and norm analysis of clinical guidelines are integrated to guide the generation of clinical pathways. Business process modeling notation best practice is adopted to enable rigorous control over the process of care by ensuring completeness, consistency, comprehensiveness and adaptability of the clinical pathway.

A unique contribution of this paper is a semiotic method for generation of clinical pathways which can addresses social and informal/safety factors which conspire together to influence the outcome of patient interaction and safety. This is achieved through modeling clinical pathways using SAM and NAM. The strength of this modeling approach results from two primary techniques, both of which are rigorous and well tested. The BPMN is a robust method that 
provides a set of techniques and notations for process modeling. SAM is a well established method for domain knowledge representation result of which is represented in ontology charts. This research offers a solution for modeling clinical activities of a CP in terms of standard business process models.

This research will serve as a basis for future studies and will be subject to rigorous validation by applying the proposed methodology to model CPs. It is also suggested that the implementation of the method if investigated in more detail in future work. We also emphasized that the initial findings of this research has been acknowledged by clinical experts from a UK hospital.

\section{References}

Abidi, S.S.R., \& Chen, H. (2006). Adaptable personalized care planning via a semantic web framework. Proceedings of the 20th Intl Cong European Fed for Medical Informatics Maastricht.

Aguilar-Saven, R.S. (2004). Business process modelling: Review and framework. International Journal of Production Economics, 90, 129-149. http://dx.doi.org/10.1016/S0925-5273(03)00102-6

Audimoolam, S., Nair, M., Gaikwad, R., \& Qing, C. (2005). The role of clinical pathways in improving patient outcomes. Retrieved 2007.

Becker, J., \& Janiesch, C. (2008). Restrictions in process design: a case study on workflows in healthcare. Business Process Management Workshops, Lecture Notes in Computer Science, 4928, 323-334. http://dx.doi.org/10.1007/978-3-540-78238-4_34

Cabitza, F., Simone, C., \& Sarini, M. (2008). Knowledge artifacts as bridges between theory and practice: the clinical pathway case. Knowledge Management in Action, 37-50. http://dx.doi.org/10.1007/978-0-387-09659-9 4

Carthey, J., Clarke, J., \& Field, C. (2010). Associate (Safer Care Priority Programme), Nhs Institute For Innovation And, Improvement 2010. Leadership for Safety: Implementing Human Factors in Healthcare.

Corrigan, J. (2005). Crossing the quality chasm. Building a Better Delivery System. Washington: The National Academy Press.

Dadam, P., Reichert, M., \& Kuhn, K. (1997). Clinical Workflows-The Killer Application for Process-oriented Information Systems? Technical Report. Universität Ulm.

Dijerome, L. (1992). The nursing case management computerized system: meeting the challenge of health care delivery through technology. Computers in nursing, 10, 250. http://dx.doi.org/10.1097/00024665-199211000-00005 
Forster, P. (2003). To err is human. Annals of human genetics, 67, 2-4. http://dx.doi.org/10.1046/j.1469-1809.2003.00002.x

Gruber, T.R. (1995). Toward principles for the design of ontologies used for knowledge sharing. International Journal of Human Computer Studies, 43, 907-928. http://dx.doi.org/10.1006/ijhc.1995.1081

Hurley, K.F., \& Abidi, S.S.R. (2007). Ontology engineering to model clinical pathways: Towards the computerization and execution of clinical pathways. IEEE, 536-541.

Indulska, M., Recker, J., Rosemann, M., \& Green, P. (2009). Business process modeling: Current issues and future challenges. Advanced Information Systems Engineering, Lecture Notes in Computer Science, 5565, 501-514. http://dx.doi.org/10.1007/978-3-642-02144-2_39

Liu, K. (2000). Semiotics in information systems engineering. Cambridge; New York: Cambridge University Press. http://dx.doi.org/10.1017/CBO9780511543364

Liu, K. (2001). Information, organisation, and technology: studies in organisational semiotics. Boston: Kluwer Academic Publishers.

Liu, K., \& Ong, T. (1999). A modelling approach for handling business rules and exceptions. The Computer Journal, 42, 221-231. http://dx.doi.org/10.1093/comjnl/42.3.221

Liu, K., Sun, L., Barjis, J., \& Dietz, J.L.G. (2003). Modelling dynamic behaviour of business organisations-extension of DEMO from a semiotic perspective. Knowledge-Based Systems, 16, 101-111. http://dx.doi.org/10.1016/S0950-7051(02)00077-1

Mor, P.C., \& Anand, K. (2012). Comparing computer-interpretable guideline models: a case study approach.

Mould, G., Bowers, J., \& Ghattas, M. (2010). The evolution of the pathway and its role in improving patient care. Quality and Safety in Health Care, 19, 1. http://dx.doi.org/10.1136/qshc.2009.032961

Muthu, S., Whitman, L., \& Cheraghi, S.H. (2006). Business process reengineering: a consolidated methodology. Proceedings of the 4th Annual International Conference on Industrial Engineering Theory, Applications, and Practice, 1999 U.S. Department of the Interior - Enterprise Architecture.

Okada, O., Ohboshi, N., Kuroda, T., Nagase, K., \& Yoshihara, H. (2005). Electronic clinical path system based on semistructured data model using personal digital assistant for onsite access. Journal of Medical Systems, 29, 379-389. http://dx.doi.org/10.1007/s10916-005-5896-8 
Ryan, A. (2006). Towards semantic interoperability in healthcare: ontology mapping from SNOMED-CT to HL7 version 3. In, 2006. Australian Computer Society, Inc., 69-74.

Sonnenberg, F. \& Hagerty, C. (2006). Computer-interpretable clinical practice guidelines. Where Are We and where Are We Going. IMIA Yearbook of Medical Informatics, 145-158.

Staccini, P., Joubert, M., Quaranta, J.F., \& Fieschi, M. (2005). Mapping care processes within a hospital: from theory to a web-based proposal merging enterprise modelling and ISO normative principles. International Journal of Medical Informatics, 74, 335-344. http://dx.doi.org/10.1016/j.ijmedinf.2004.07.003

Stamper, R. (2009). The Chemistry of Society: Organisational Semiotics as an Empirical Social Science. Proceedings of the 11th International Conference in Informatics and Semiotics in Organisations, an IFIP WG8.1 Working Conference. Beijing, China.

Stamper, R.K. (2001). Organisational semiotics: Informatics without the computer. Information, organisation and technology: Studies in organisational semiotics, 115-171.

West, M.A., Guthrie, J.P., Dawson, J.F., Borrill, C.S., \& Carter, M. (2006). Reducing patient mortality in hospitals: The role of human resource management. Journal of Organizational Behavior, 27, 983-1002. http://dx.doi.org/10.1002/job.396

Wright, G.H. (1963). Norm and action: a logical enquiry. Humanities Press.

Yang, H., Liu, K., \& Li, W. (2010). Adaptive Requirement-Driven Architecture for Integrated Healthcare Systems. Journal of Computers, 5, 186-193. http://dx.doi.org/10.4304/jcp.5.2.186-193

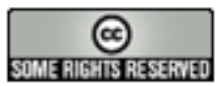

El artículo está con Reconocimiento-NoComercial 3.0 de Creative Commons. Puede copiarlo, distribuirlo y comunicarlo públicamente siempre que cite a su autor y a Intangible Capital. No lo utilice para fines comerciales. La licencia completa se puede consultar en http://creativecommons.org/licenses/by-nc/3.0/es/ 